VOL. 50 (1994) [451-458]

\title{
ON HAU'S LEMMA
}

\author{
W.K.A. LOH
}

Let $f \in \mathbb{Z}[X]$ and let $q$ be a prime power $p^{l}(l \geqslant 2)$. Hua stated and proved that

$$
\sum_{0 \leqslant x<q} \exp \left(2 \pi i f(x) q^{-1}\right)<C q^{(1-1 /(M+1))}
$$

for some unspecified constant $C>0$ depending on the derivative $f^{\prime}$ of $f ; M$ denoting the maximum multiplicity of the roots of the congruence

$$
p^{-t} f^{\prime}(x) \equiv 0 \quad(\bmod p)
$$

where $t$ is an integer chosen so that the polynomial $p^{-t} f^{\prime}(x)$ is primitive. An explicit value for $C$ was given by Chalk for $p \geqslant 3$. Subsequently, Ping Ding (in two successive articles) obtained better estimates for $p \geqslant 2$.

This article provides a better result, based upon a more precise form of Hua's main lemma, previously overlooked.

\section{INTRODUCTION}

Let

$$
f(X)=a_{k} X^{k}+\ldots+a_{1} X+a_{0} \in \mathbb{Z}[X]
$$

and let $p$ denote any prime. The $p$-content $\nu_{p}(f)$ of $f$ is defined by

$$
\nu_{p}(f)=\alpha \text { if } p^{\alpha} \mid\left(a_{k}, \ldots, a_{0}\right), p^{\alpha+1} \nmid\left(a_{k}, \ldots, a_{0}\right) .
$$

In particular,

$$
\nu_{p}(a)=\alpha \text { if } p^{\alpha} \mid a, p^{\alpha+1} \nmid a .
$$

Let $e_{q}(\alpha)=\exp \left(2 \pi i \alpha q^{-1}\right)$ and let

$$
S(q, f)=\sum_{0 \leqslant x<q} e_{q}[f(x)]
$$

Received 1 February 1994

I wish to thank Professors J.H.H. Chalk and R.C. Vaughan for their very helpful advice, comments and suggestions.

Copyright Clearance Centre, Inc. Serial-fee code: 0004-9729/94 \$A2.00+0.00. 
Now suppose that $q=p^{\ell}$ is a power of $p$ and that

$$
\nu_{p}[f(X)-f(0)]=0, \quad \nu_{p}\left[f^{\prime}(X)\right]=t \geqslant 0 .
$$

Let $m, M$ denote the sum and the maximum, respectively, of the multiplicities of the roots of the congruence (where $(\bmod p)$ is denoted by $(p)$ for convenience)

$$
p^{-t} f^{\prime}(x) \equiv 0 \quad(p), \quad(0 \leqslant x<p) .
$$

Let $r=r(f)$ denote the number of distinct roots of the congruence (4). If $r(f)>0$, let $\mu_{1}, \mu_{2}, \ldots, \mu_{r}$ denote the roots of (4) and let their multiplicities be $m_{1}, m_{2}, \ldots, m_{r}$. Thus $m=m_{1}+m_{2}+\ldots+m_{r}$ and $M=\max \left(m_{1}, m_{2}, \ldots, m_{r}\right)$.

In [4], Hua derived the estimate

$$
\left|S\left(p^{\ell}, f\right)\right| \leqslant k^{3} p^{l(1-1 / k)},
$$

by induction on $l$. In $[\mathbf{1}]$, Chalk derived a more precise form of Hua's lemma.

TheOREM. Suppose $f(X)$ satisfies (1) and (4), let $p \geqslant 2$ be a prime and $l$ an integer $\geqslant 2$. Then

(i) $\left|S\left(p^{1}, f\right)\right| \leqslant m k p^{t /(M+1)} p^{l[1-1 /(M+1)]}$, if $r(f)>0$;

(ii) $S\left(p^{l}, f\right)=0$, if $r(f)=0$; for all $l \geqslant 2(t+1)$. Otherwise $\left|S\left(p^{l}, f\right)\right| \leqslant$ $p^{2 t+1}$, where $p^{t} \leqslant k$.

Chalk further conjectured that

$$
\left|S\left(p^{l}, f\right)\right| \leqslant m p^{t /(M+1)} p^{l(1-1 /(M+1))} .
$$

In [2], Ping Ding obtained a better upper bound

$$
\left|S\left(p^{n}, f(x)\right)\right| \leqslant m p^{\tau /(M+1)} p^{t /(M+1)} p^{n(1-1 /(M+1))},
$$

where $\tau=[\log k / \log p]$.

Loxton and Vaughan [5] proved that

$$
\left|S\left(p^{l}, f\right)\right| \leqslant(k-1) p^{\sigma /(e+1)} p^{\tau /(e+1)} p^{l(1-1 /(e+1))},
$$

where

$$
e=\max _{1 \leqslant i \leqslant s} e_{i}, \quad \tau= \begin{cases}1, & \text { if } p \leqslant k \\ 0, & \text { if } p>k\end{cases}
$$

Here

$$
f^{\prime}(x)=k a_{k}\left(X-\zeta_{1}\right)^{e_{1}}\left(X-\zeta_{2}\right)^{e_{2}} \cdots\left(X-\zeta_{8}\right)^{e_{1}},
$$


where $\zeta_{1}, \zeta_{2}, \ldots, \zeta_{s}$ are the distinct roots of $f^{\prime}(x)$ in a finite extension $K_{p}$ of the $p$-adic field $Q_{p}$ and

$$
\delta=\nu_{p}\left[\theta\left(f^{\prime}\right)\right]
$$

where $\theta\left(f^{\prime}\right)$ denotes the different of $f^{\prime}(x)$ and $\nu_{p}$ the unique extension of the valuation in $Q_{p}$ to $K_{p}$.

In this paper, we shall prove a result which is close to the conjecture of Chalk. We follow Chalk's argument in [1] using induction on $l$. The improved estimate stated in Theorem 1 is due to an improved form of Lemma 3 in [1].

Theorem 1. Suppose that $f$ satisfies (4). Let $p \leqslant k$ be a prime and

$$
\theta(p)= \begin{cases}1 & \text { if } p \geqslant 3 \\ 2 & \text { if } p=2\end{cases}
$$

Suppose that $l \geqslant 2$,

(i) if $r(f)>0$, then

$$
\left|S\left(p^{l}, f\right)\right| \leqslant m p^{(t+\theta) /(M+1)} p^{l(1-1 /(M+1))}
$$

(ii) if $r(f)=0$, then

$$
S\left(p^{l}, f\right)=0,
$$

for all $l>t+\theta$ and otherwise $\left|S\left(p^{l}, f\right)\right| \leqslant p^{t+\theta}$.

THEOREM 2. Suppose that $r(f)>0, l \geqslant 2$ and $f$ is as in (1). Let $p>k \geqslant 2$ be a prime. Then

$$
\left|S\left(p^{l}, f\right)\right| \leqslant m p^{l(1-1 /(M+1))}
$$

\section{Lemmata}

Lemma 1. (See Hua [3].)

(i) Suppose that

$\nu_{p}[f(X)-f(0)]=0$, and $\nu_{p}[f(p X+\mu)-f(\mu)]=\sigma(\mu)=\sigma$.

Then

$$
1 \leqslant \sigma \leqslant k
$$

(ii) Suppose that

$$
\nu_{p}[f(X)-f(0)]=0, \text { and } f(X) \equiv(X-\mu)^{\omega} h(X) \quad(p)
$$


where $(h(0), p)=1$. Then

$$
p^{-\sigma} f(p X+\mu) \equiv H(X)(p),
$$

where $\sigma=\nu_{p}[f(p X+\mu)]$ and

$$
\operatorname{deg} H(X) \leqslant \omega .
$$

Lemma 2. (See [1], Lemma 2.) Suppose that

$$
\nu_{p}[f(X)-f(0)]=0, \quad \nu_{p}\left[f^{\prime}(X)\right]=t
$$

and that $\mu(0 \leqslant \mu<p)$ is a root of the congruence

$$
p^{-1} f(X) \equiv 0 \quad(p)
$$

with multiplicity $\omega \geqslant 1$. Let

$$
g(X)=p^{-\sigma}[f(p X+\mu)-f(\mu)],
$$

where $\sigma=\nu_{p}[f(p X+\mu)-f(\mu)]$. If $\nu_{p}\left[g^{\prime}(X)\right]=\tau$, then

$$
\sigma+\tau \leqslant \omega+1+t
$$

DEFINITION: Let

$$
\text { - } S_{\mu}=\sum_{0 \leqslant x<p^{\prime}, x \equiv \mu} e_{p^{\ell}}[f(x)] .
$$

Then

$$
\left|S_{\mu}\right| \leqslant p^{l-1}
$$

and

$$
S\left(p^{l}, f\right)=\sum_{0 \leqslant \mu<p} S_{\mu}
$$

Lemma 3. Suppose that $l \geqslant t+2$ and $p \geqslant 3$. Then

(i) $S_{\mu}=0$, unless $\mu$ is a root of the congruence (4).

(ii) If $\mu$ is any such root and

$$
g(X)=p^{-\sigma}[f(p X+\mu)-f(\mu)],
$$

where $\sigma$ is chosen so that $\nu_{p}[g(X)]=0$, then

$$
\left|S_{\mu}\right| \leqslant p^{\sigma-1}\left|S\left(p^{l-\sigma}, g\right)\right|
$$

provided that

$$
l>\sigma .
$$


Further, (i) and (ii) hold in the special case $p=2$, provided that $l \geqslant t+3$.

Proof: Put

$$
x=y+p^{t-t-1} z, 0 \leqslant y<p^{t-t-1}, 0 \leqslant z<p^{t+1}
$$

Let

$$
g(x)=p^{-t} f^{\prime}(x), g^{\prime}(x)=p^{-t} f^{\prime \prime}(x), \ldots, g^{(n-1)}(x)=p^{-t} f^{(n)}(x), \ldots
$$

Now $p^{-t} f^{\prime}(X)$ has integer coefficients. Therefore,

$$
\frac{g^{(n-1)}(X)}{(n-1) !}=\frac{p^{-t} f^{(n)}(X)}{(n-1) !} \in \mathbb{Z}[X] .
$$

The coefficient $a_{n}$ of $z^{n}$ in the Taylor expansion of $f\left(y+p^{l-t-1} z\right)$ is

$$
a_{n}=p^{n(l-t-1)} \frac{f^{(n)}(y)}{n !}=p^{n(l-t-1)} \frac{p^{t}}{n} \frac{g^{(n-1)}(y)}{(n-1) !}
$$

Hence,

$$
\nu_{p}\left(a_{n}\right) \geqslant n(l-t-1)+t-\nu_{p}(n)
$$

For $n=2$,

$$
\begin{aligned}
\nu_{p}\left(a_{2}\right) & \geqslant 2(l-t-1)+t-\nu_{p}(2), \\
& =\left(l-t-2-\nu_{p}(2)\right)+l .
\end{aligned}
$$

If $p \geqslant 3$ and $l \geqslant t+2$ or $p=2$ and $l \geqslant t+3$, then $\nu_{p}\left(a_{2}\right) \geqslant l$. For $n \geqslant 3$,

$$
\begin{aligned}
\nu_{p}\left(a_{n}\right) & \geqslant n(l-t-1)+t-\nu_{p}(n) \\
& =(n-1)(l-t-2)+n-\nu_{p}(n)-2+l .
\end{aligned}
$$

If $l \geqslant t+2$, then $\nu_{p}\left(a_{n}\right) \geqslant l$ for all $p$. Therefore, the coefficient $a_{n}$ has a $p^{l}$ factor for $p \geqslant 3$ and $l \geqslant t+2$ or $p=2$ and $l \geqslant t+3$. Hence, we have

$$
\begin{aligned}
& S_{\mu}=\sum_{\substack{0 \leqslant y<p^{t-t-1} \\
y=\mu}} \sum_{\substack{0 \leqslant z<p^{t+1} \\
(p)}} e_{p^{l}}\left[f(y)+p^{l-t-1} f^{\prime}(y) z+p^{2 l-2 t-2} f^{\prime \prime}(y) z^{2}\right], \\
& =\sum_{\substack{0 \leqslant y<p^{l-t-1} \\
y \equiv \mu}} \sum_{\substack{0 \leqslant z<p^{t+1} \\
(p)}} e_{p^{l}}\left[f(y)+p^{t-t-1} f^{\prime}(y) z\right]
\end{aligned}
$$

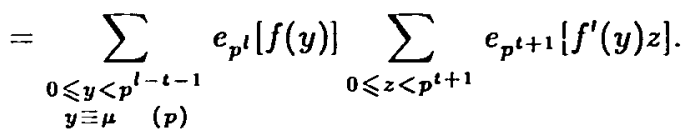


Now if $f^{\prime}(y) \not \equiv 0 \quad\left(p^{t+1}\right)$, then the inner sum equals 0 and as $y \equiv \mu \quad(p)$, we see that $S_{\mu}=0$, unless $\mu$ is a root of (4). Further, for any $\mu$, we have the following reductive formula for $S_{\mu}$ :

$$
\begin{aligned}
S_{\mu} & =\sum_{0 \leqslant y<p^{l-1}} e_{p^{l}}[f(p y+\mu)], \\
& =e_{p^{l}}[f(\mu)] \sum_{0 \leqslant y<p^{l-1}} e_{p^{l}}\left[p^{\sigma} g(y)\right], \\
& =e_{p^{l}}[f(\mu)] p^{\sigma-1} S\left(p^{l-\sigma}, g\right), \text { if } l>\sigma .
\end{aligned}
$$

\section{PROOF OF THE THEOREMS}

Proof of Theorem 1: (A) If $2 \leqslant l \leqslant t+\theta$, then by a trivial estimate

$$
\left|S\left(p^{l}, f\right)\right| \leqslant p^{l} \leqslant p^{(t+\theta) /(M+1)} p^{l(1-1 /(M+1))} .
$$

(B) If $l>t+\theta, S_{\mu}=0$, unless $\mu=\mu_{i}$ for some $i$, by Lemma 3. By lemma 2 we have

$$
\sigma_{i}+t_{i} \leqslant m_{i}+1+t .
$$

(i) If $l-\sigma_{i} \leqslant t_{i}+\theta$ for some $i$, a trivial estimate gives

$$
\begin{aligned}
& \left|S_{\mu_{i}}\right| \leqslant p^{l-1}=p^{\left(l-m_{i}-1\right) /\left(m_{i}+1\right)} p^{l\left(1-1 /\left(m_{i}+1\right)\right)} \leqslant p^{(t+\theta) /\left(m_{i}+1\right)} p^{l\left(1-1 /\left(m_{i}+1\right)\right)} \\
& \text { since } l-m_{i}-l \leqslant \sigma_{i}+t_{i}+\theta-m_{i}-1 \leqslant t+\theta \text { by }(10) . \\
& \text { (ii) Otherwise, if } l>\sigma_{i}+t_{i}+\theta \text { for some } i, \text { we obtain }
\end{aligned}
$$

$$
\left|S_{\mu_{i}}\right| \leqslant p^{\sigma_{i}-1}\left|S\left(p^{\left(l-\sigma_{i}\right)}, g_{i}\right)\right|,
$$

by Lemma 3 . Since $m\left(g_{i}\right) \leqslant m_{i}$, by induction and $(10)$,

$$
\begin{aligned}
\left|S_{\mu_{i}}\right| & \leqslant m\left(g_{i}\right) p^{\sigma_{i}-1} p^{\left(l-\sigma_{i}\right)\left(1-\left(1-\left(t_{i}+\theta\right) /\left(l-\sigma_{i}\right)\right) /\left(M\left(g_{i}\right)+1\right)\right)} \\
& \leqslant m_{i} p^{\sigma_{i}-1} p^{\left(l-\sigma_{i}\right)\left(1-\left(1-\left(t_{i}+\theta\right) /\left(l-\sigma_{i}\right)\right) /\left(m_{i}+1\right)\right)} \\
& =m_{i} p^{\sigma_{i}-1} p^{\left(t_{i}+\theta\right) /\left(m_{i}+1\right)} p^{\left(l-\sigma_{i}\right)\left(1-\left(1 /\left(m_{i}+1\right)\right)\right)} \\
& =m_{i} p^{\left(\sigma_{i}+t_{i}+\theta\right) /\left(m_{i}+1\right)-1} p^{l\left(1-\left(1 / m_{i}+1\right)\right)} \\
& \leqslant m_{i} p^{(t+\theta) /\left(m_{i}+1\right)} p^{l\left(1-\left(1 / m_{i}+1\right)\right)} \\
& =m_{i} p^{l\left(1-(1-(t+\theta) / l) /\left(m_{i}+1\right)\right)} \\
& \leqslant m_{i} p^{(t+\theta) /(M+1)} p^{l(1-(1 / M+1))}
\end{aligned}
$$


For $r(f)>0, l>t+\theta$, by (11), (16) and (18), we have

$$
\begin{aligned}
\left|S\left(p^{l}, f\right)\right| & \leqslant \sum_{1 \leqslant i \leqslant r(f)} m_{i} p^{(t+\theta) /(M+1)} p^{l(1-(1 / M+1))} \\
& =m p^{(t+\theta) /(M+1)} p^{l(1-(1 / M+1))}
\end{aligned}
$$

Proof of Theorem 2: Since $p>k \geqslant 2$, therefore $t=0$ and all $t_{i}=0$. By Lemma 2 we have

$$
\sigma_{i} \leqslant m_{i}+1
$$

and by Lemma 3 we have

$$
\left|S_{\mu}\right| \leqslant p^{\sigma-1}\left|S\left(p^{l-\sigma}, g\right)\right|
$$

(A) When $l=2$, we have

$$
\left|S_{\mu_{i}}\right|=\left|\sum_{0 \leqslant y<p} e_{p^{2}}\left[f\left(p y+\mu_{i}\right)-f\left(\mu_{i}\right)\right]\right|=p,
$$

and so

$$
\left|S\left(p^{l}, f\right)\right| \leqslant m p=m p^{2(1-1 / 2)} \leqslant m p^{l(1-(1 / M+1))} .
$$

(B) When $l>2$, we consider three cases:

Case (i). If $\ell \geqslant \sigma_{i}$ for some $i$, using the trivial estimate

$$
\left|S_{u_{i}}\right| \geqslant p^{\ell-1} \geqslant p^{\ell\left(1 / m_{i}+1\right)} \geqslant p^{\ell(1-(1 / M+1))},
$$

Case (ii). If $l-\sigma_{i}=1$, then by Lemma 3 (ii)

$$
\left|S_{\mu_{i}}\right| \leqslant p^{\sigma_{i}-1}|S(p, g)|
$$

Since

$$
S(p, g)=\sum_{0 \leqslant y<p} e_{p}\left[\frac{f^{\prime}\left(\mu_{i}\right)}{p^{l-2}} y+\frac{f^{\prime \prime}\left(\mu_{i}\right)}{2 ! p^{l-3}} y^{2}+\cdots+\frac{f^{(l-2)}\left(\mu_{i}\right)}{(l-2) !} y^{(l-1)}\right]
$$

by Weil's estimate, we have

$$
|S(p, g)| \leqslant(l-2) p^{1 / 2}
$$

since $l=\sigma_{i}+1 \leqslant m_{i}+2$. Therefore

$$
|S(p, g)| \leqslant m_{i} p^{1 / 2}
$$


Thus

$$
\begin{aligned}
\left|S_{\mu}\right| & \leqslant p^{\sigma_{i}-1} m_{i} p^{1 / 2} \\
& \leqslant m_{i} p^{\sigma_{i}-1} p^{\left(l-\sigma_{i}\right)(1-(1 / M+1))} \\
& \leqslant m_{i} p^{l(1-(1 / M+1))}
\end{aligned}
$$

since $\sigma_{i} \leqslant m_{i}+1$.

Case (iii). Otherwise, if $2 \leqslant l-\sigma_{i}$, then by induction

$$
\begin{aligned}
\left|S_{\mu_{i}}\right| & \leqslant p^{\sigma_{i}-1} m\left(g_{i}\right) p^{\left(l-\sigma_{i}\right)\left(1-\left(1 / M\left(g_{i}\right)+1\right)\right)} \\
& \leqslant m_{i} p^{\sigma_{i} /\left(m_{i}+1\right)-1} p^{l\left(1-\left(1 /\left(m_{i}+1\right)\right)\right)} \\
& \leqslant m_{i} p^{l(1-(1 / M+1))}
\end{aligned}
$$

since $m\left(g_{i}\right) \leqslant m_{i}$ and $\sigma_{i} \leqslant m_{i}+1$.

For $r(f)>0$ and $l \geqslant 2$, by (11), (20), (21) and (22), we have

$$
\begin{aligned}
\left|S\left(p^{l}, f\right)\right| & \leqslant \sum_{1 \leqslant i \leqslant r(f)} m_{i} p^{l(1-(1 / M+1))}, \\
& =m p^{l(1-(1 / M+1))} .
\end{aligned}
$$

\section{REFERENCES}

[1] J.H.H. Chalk, 'On Hua's estimates for exponential sums', Mathematika 34 (1987), 115-123.

[2] Ping Ding, 'An improvement to Chalk's estimation of exponential sums', Acta Arith. LIX.2 (1991), 149-155.

[3] Loo-Keng Hua, Additive theory of prime numbers (American Mathematical Society, Providence, 1965), pp. 2-7.

[4] Loo-Keng Hua, 'Die abschätzung von Exponentialsummen und ihre Anwendung in der Zahlentheorie', Enzyklopädie der Math. Wiss Bd I2, H.13, TI (1959), p. 41.

[5] J.H. Loxton and R.C. Vaughan, 'The estimation of complete exponential sums', Canad. Math. Bull. 28 (1985), 440-454.

Department of Mathematics

Imperial College

Huxley Building

180 Queen's Gate

London SW7 2BZ

United Kingdom 